HUB-EP-96/39

\title{
Perturbative renormalisation of bilinear quark and gluon operators*
}

\author{
M. Göckeler ${ }^{\mathrm{a}, \mathrm{b}, \mathrm{c}}$, R. Horsley ${ }^{\mathrm{d}}$, E.-M. Ilgenfritz ${ }^{\mathrm{d}}$, H. Oelrich ${ }^{\mathrm{a}, \mathrm{e}}$, H. Perlt ${ }^{\mathrm{f}}$, P. Rakow $^{\mathrm{a}, \mathrm{e}}$, G. Schierholz ${ }^{\mathrm{a}, \mathrm{g}}$, \\ A. Schiller ${ }^{f}$ and P. Stephenson ${ }^{\mathrm{e}}$ \\ a HLRZ, c/o Forschungszentrum Jülich, D-52425 Jülich, Germany \\ b Institut für Theoretische Physik, RWTH Aachen, D-52056 Aachen, Germany \\ c Institut für Theoretische Physik, J. W. Goethe Universität, D-60054 Frankfurt, Germany \\ d Institut für Physik, Humboldt-Universität zu Berlin, D-10115 Berlin, Germany \\ e DESY-IfH Zeuthen, D-15735 Zeuthen, Germany \\ f Institut für Theoretische Physik, Universität Leipzig, D-04109 Leipzig, Germany \\ g DESY, D-22603 Hamburg, Germany
}

The renormalisation constants for local bilinear quark operators are calculated using the Sheikholeslami-Wohlert improved action. In addition we compute the renormalisation constant of the leading gluon operator for different group representations and discuss the mixing of the operators $\vec{E}^{2}$ and $\vec{B}^{2}$.

\section{INTRODUCTION}

Forward hadron matrix elements of bilinear quark and gluon operators, as they appear e.g. in the operator product expansion for the nucleon structure functions, have been calculated with some success on the lattice [1]. In order to extract the relevant physical information from the lattice matrix elements one has to renormalise the operators. This can be done perturbatively [2 5] or non-perturbatively [6,77]. Both methods have their advantages and disadvantages. In this paper we present a one-loop calculation of the renormalisation constants of some bilinear quark operators for improved Wilson fermions and of the gluon operator $\operatorname{Tr} F_{\mu \rho} F_{\rho \nu}$. All calculations have been performed in the quenched approximation and for zero renormalised quark mass.

\footnotetext{
${ }^{*}$ Contribution presented by H. Perlt
}

\section{LOCAL QUARK OPERATORS}

Lattice calculations with Wilson fermions suffer from cut-off effects of $\mathcal{O}(a)$, where $a$ is the lattice spacing. By adding the local counterterm

$$
\Delta S=-i \mathrm{c}_{\mathrm{sw}} g a^{4} \sum_{n, \mu \nu} \frac{a r}{4} \bar{\psi}_{n} \sigma_{\mu \nu} F_{n, \mu \nu} \psi_{n}
$$

to the action, cut-off effects in on-shell quantities can be reduced to $\mathcal{O}\left(a^{2}\right)$ [8 for a particular choice of $\mathrm{c}_{\mathrm{sw}}$.

In hadron matrix element calculations one has to improve the operators as well. This again can be achieved by adding certain counterterms. For a class of operators, in particular for the operators considered in this paper, the counterterms can be obtained by a rotation of the fermion fields

$\bar{\psi} \rightarrow \bar{\psi}\left(1+\mathrm{c}_{\mathrm{swr}} \frac{a r}{4} \overleftarrow{D}\right), \quad \psi \rightarrow\left(1-\mathrm{c}_{\mathrm{swr}} \frac{a r}{4} \vec{D}\right) \psi$ with $\mathrm{c}_{\mathrm{swr}}$ to be determined properly.

In lowest order perturbation theory $\mathrm{c}_{\mathrm{sw}}=$ $\mathrm{c}_{\mathrm{swr}}=1$. A non-perturbative evaluation of $\mathrm{c}_{\mathrm{sw}}$ 
was given in 97. Throughout the rest of the paper we shall assume $r=1$

We consider the operators

$\mathcal{O}=\bar{\psi} \Gamma \psi$

with

$\Gamma=1, \gamma_{5}, \gamma_{\mu}, \gamma_{\mu} \gamma_{5}, \sigma_{\mu \nu} \gamma_{5}$

The renormalisation constants are generically defined by (for details see [2])

$\mathcal{O}(\mu)=Z_{\mathcal{O}}(a \mu, g) \mathcal{O}(a)$,

$\langle q(p)|\mathcal{O}(\mu)| q(p)\rangle=\left.\langle q(p)|\mathcal{O}(a)| q(p)\rangle\right|_{p^{2}=\mu^{2}} ^{\text {tree }}$.

They can be cast into the form

$Z_{\mathcal{O}}=1-\frac{g^{2}}{16 \pi^{2}} C_{F}\left(\gamma_{\mathcal{O}} \ln (a \mu)+B_{\mathcal{O}}\right), C_{F}=\frac{4}{3}$,

where $\gamma_{\mathcal{O}}$ is the anomalous dimension and $B_{\mathcal{O}}$ is the finite part of the renormalisation constant. The definition (2) corresponds to the momentum subtraction scheme.

$B_{\mathcal{O}}$ receives contributions from two different sources: the proper operator radiative corrections and the self-energy diagrams. We write accordingly

$B_{\mathcal{O}}=B_{\mathcal{O}}^{\text {proper }}+B_{\mathcal{O}}^{\text {self }}$.

Using the procedure outlined in [2] we obtain ?

$$
\begin{aligned}
B_{1}^{\text {proper }}= & 6.100-19.172 \mathrm{c}_{\mathrm{swr}}-4.981 \mathrm{c}_{\mathrm{swr}}{ }^{2}+ \\
& 9.987 \mathrm{c}_{\mathrm{sw}}+13.801 \mathrm{c}_{\mathrm{swr}} \mathrm{c}_{\mathrm{sw}}+ \\
& 2.177 \mathrm{c}_{\mathrm{swr}}{ }^{2} \mathrm{c}_{\mathrm{sw}}+0.017 \mathrm{c}_{\mathrm{sw}}{ }^{2}- \\
& 3.538 \mathrm{c}_{\mathrm{swr}} \mathrm{c}_{\mathrm{sw}}{ }^{2}-0.288 \mathrm{c}_{\mathrm{swr}}{ }^{2} \mathrm{c}_{\mathrm{sw}}{ }^{2}+ \\
& \left(-2+\gamma_{E}-F_{0}\right) \xi, \\
B_{\gamma_{5}}^{\text {proper }}= & 15.743+8.660 \mathrm{c}_{\mathrm{swr}}{ }^{2}- \\
& 5.715 \mathrm{c}_{\mathrm{swr}}{ }^{2} \mathrm{c}_{\mathrm{sw}}+3.433 \mathrm{c}_{\mathrm{sw}}{ }^{2}+ \\
& 1.361 \mathrm{c}_{\mathrm{swr}}{ }^{2} \mathrm{c}_{\mathrm{sw}}{ }^{2}+ \\
& \left(-2+\gamma_{E}-F_{0}\right) \xi, \\
B_{\gamma_{\mu}}^{\text {proper }}= & 8.765-9.786 \mathrm{c}_{\mathrm{swr}}+3.525 \mathrm{c}_{\mathrm{swr}}{ }^{2}- \\
& 2.497 \mathrm{c}_{\mathrm{sw}}+3.416 \mathrm{c}_{\mathrm{swr}} \mathrm{c}_{\mathrm{sw}}-
\end{aligned}
$$

${ }^{2}$ The numbers are given here only to three decimal places but are known to more than ten places.

$$
\begin{aligned}
& 1.973 \mathrm{c}_{\mathrm{swr}}{ }^{2} \mathrm{c}_{\mathrm{sw}}+0.854 \mathrm{c}_{\mathrm{sw}}{ }^{2}+ \\
& 0.885 \mathrm{c}_{\mathrm{swr}} \mathrm{c}_{\mathrm{sw}}{ }^{2}+0.412 \mathrm{c}_{\mathrm{swr}}{ }^{2} \mathrm{c}_{\mathrm{sw}}{ }^{2}+ \\
B_{\gamma_{\mu} \gamma_{5}}^{\text {proper }}= & \left(-1+\gamma_{E}-F_{0}\right) \xi, \\
& 3.944-19.372 \mathrm{c}_{\mathrm{swr}}-3.296 \mathrm{c}_{\mathrm{swr}}{ }^{2}+ \\
& 2.497 \mathrm{c}_{\mathrm{sw}}+10.317 \mathrm{c}_{\mathrm{swr}} \mathrm{c}_{\mathrm{sw}}+ \\
& 1.973 \mathrm{c}_{\mathrm{swr}}{ }^{2} \mathrm{c}_{\mathrm{sw}}-0.854 \mathrm{c}_{\mathrm{sw}}{ }^{2}- \\
& 0.885 \mathrm{c}_{\mathrm{swr}} \mathrm{c}_{\mathrm{sw}}{ }^{2}-0.412 \mathrm{c}_{\mathrm{swr}}{ }^{2} \mathrm{c}_{\mathrm{sw}}{ }^{2}+ \\
B_{\sigma_{\mu \nu} \gamma_{5}}^{\text {proper }}= & \left(-1+\gamma_{E}-F_{0}\right) \xi, \\
& 1.166-16.243 \mathrm{c}_{\mathrm{swr}}-0.461 \mathrm{c}_{\mathrm{swr}}{ }^{2}- \\
& 0.564 \mathrm{c}_{\mathrm{sw}}+6.855 \mathrm{c}_{\mathrm{swr}} \mathrm{c}_{\mathrm{sw}}+ \\
& 0.590 \mathrm{c}_{\mathrm{swr}}-0.575 \mathrm{c}_{\mathrm{sw}}{ }^{2}+0.179 \mathrm{c}_{\mathrm{swr}}{ }^{2} \mathrm{c}_{\mathrm{sw}}{ }^{2}+ \\
& \left(\gamma_{E}-F_{0}\right) \xi .
\end{aligned}
$$

Here $\xi$ is the gauge parameter $(\xi=0$ for Feynman gauge, $\xi=1$ for Landau gauge) and $F_{0}=$ 4.369225. The self-energy contribution is

$$
\begin{aligned}
B_{\mathcal{O}}^{\text {self }}= & -0.381-2.249 \mathrm{c}_{\mathrm{sw}}-1.397 \mathrm{c}_{\mathrm{sw}}{ }^{2}+ \\
& 8 \pi^{2} Z_{0}+\left(1-\gamma_{E}+F_{0}\right) \xi,
\end{aligned}
$$

where $Z_{0}=0.154933$.

Since the Wilson coefficients are usually computed in the MS or $\overline{\mathrm{MS}}$ scheme, one would like to know the renormalisation constants in these schemes too. The transformations between the different schemes are [2]

$B_{\mathcal{O}}^{M S}=B_{\mathcal{O}}-B_{\mathcal{O}}^{c o n}$,

$B_{\mathcal{O}}^{\overline{M S}}=B_{\mathcal{O}}^{M S}+\frac{\gamma_{\mathcal{O}}}{2}\left(\gamma_{E}-\ln 4 \pi\right)$,

where $\gamma_{\mathcal{O}}$ and $B_{\mathcal{O}}^{c o n}$, the finite contribution of the continuum integrals to $B_{\mathcal{O}}$, are given in the table below:

\begin{tabular}{||c|c|c||}
\hline $\mathcal{O}$ & $\gamma_{\mathcal{O}}$ & $B_{\mathcal{O}}^{\text {con }}$ \\
\hline $1, \gamma_{5}$ & -6 & $5+\frac{\gamma_{\mathcal{O}}}{2} c_{E}-\xi$ \\
$\gamma_{\mu}, \gamma_{\mu} \gamma_{5}$ & 0 & 0 \\
$\sigma_{\mu \nu} \gamma_{5}$ & 2 & $-1+\frac{\gamma_{\mathcal{O}}}{2} c_{E}+\xi$ \\
\hline
\end{tabular}

Here $c_{E}=\gamma_{E}-\ln 4 \pi$. In the MS and in the $\overline{\mathrm{MS}}$ scheme $B_{\mathcal{O}}$ is gauge independent, as one can easily see. 
Our results for $\mathrm{c}_{\mathrm{sw}}=1, \mathrm{c}_{\mathrm{swr}}=0$ and $\mathrm{c}_{\mathrm{sw}}=$ $\mathrm{c}_{\mathrm{swr}}=1$ agree with those of [10] and [1]], respectively.

\section{GLUON OPERATOR}

Several information about the gluon content of the nucleon can be derived from the matrix element of the operator 12 ]

$\mathcal{O}_{\mu \nu}=\operatorname{Tr} F_{\mu \rho} F_{\rho \nu}$

The renormalisation constants of certain irreducible representations of (3) have been given elsewhere 13 15. Here we are interested in the operators

$\operatorname{Tr} \vec{E}^{2}=-\mathcal{O}_{44}, \operatorname{Tr} \vec{B}^{2}=\frac{1}{2}\left(\mathcal{O}_{44}-\sum_{i=1}^{3} \mathcal{O}_{i i}\right)$,

where

$\mathrm{E}_{i}=\mathrm{F}_{i 4}, \mathrm{~B}_{i}=\frac{1}{2} \epsilon_{i j k} \mathrm{~F}_{j k}$

It is clear from group theoretical arguments [16] that $\operatorname{Tr} \vec{E}^{2}$ and $\operatorname{Tr} \vec{B}^{2}$ mix under renormalisation. We write in symbolic form

$\begin{aligned} \operatorname{Tr} \vec{E}_{R}^{2} & =Z_{E E} \operatorname{Tr} \vec{E}_{0}^{2}+Z_{E B} \operatorname{Tr} \vec{B}_{0}^{2} \\ \operatorname{Tr} \vec{B}_{R}^{2} & =Z_{B E} \operatorname{Tr} \vec{E}_{0}^{2}+Z_{B B} \operatorname{Tr} \vec{B}_{0}^{2} .\end{aligned}$

In the quenched approximation all $Z$ 's are finite, i.e. have no logarithmic contributions. Symmetry arguments lead to the following relations:

$Z_{E E}=Z_{B B}, Z_{E B}=Z_{B E}$.

Making use of some of the results of [13] we obtain in the MS scheme

$Z_{E E}=Z_{B B}=1+0.123128 g^{2}$

$Z_{E B}=Z_{B E}=-0.096655 g^{2}$.

There is a combination of $\operatorname{Tr} \vec{E}^{2}$ and $\operatorname{Tr} \vec{B}^{2}$ which belongs to an irreducible representation of the hypercubic group and does not mix:

$$
\begin{aligned}
\mathcal{O}_{b} & =\mathcal{O}_{44}-\frac{1}{3}\left(\mathcal{O}_{11}+\mathcal{O}_{22}+\mathcal{O}_{33}\right) \\
& =\frac{2}{3}\left(\operatorname{Tr} \vec{B}^{2}-\operatorname{Tr} \vec{E}^{2}\right)
\end{aligned}
$$

For the renormalisation constant of this operator we find

$$
Z_{\mathcal{O}_{b}}=1+0.219783 g^{2}
$$

which confirms numerically the relation between the $Z$ 's,

$Z_{\mathcal{O}_{b}}=Z_{E E}-Z_{E B}$.

The renormalization constant $Z_{\mathcal{O}_{b}}$ should be compared with the renormalisation constant of the operator

$\mathcal{O}_{a}=\mathcal{O}_{\{i 4\}}=\operatorname{Tr}(\vec{E} \times \vec{B})_{i}$

which turns out to be 14,15]

$Z_{\mathcal{O}_{a}}=1+0.27075 g^{2}$.

(The lattice value is $1+0.29608 g^{2}$ for $\xi=0$.) The mall difference of $Z_{\mathcal{O}_{a}}$ and $Z_{\mathcal{O}_{b}}$ is due to non- $O(4)$ invariant contributions.

\section{REFERENCES}

1. M. Göckeler, this conference

2. M. Göckeler et al., Nucl. Phys. B472 (1996) 309, and references therein

3. G. Rossi, this conference

4. S. Capitani, this conference

5. A. Pochinsky, this conference

6. G. Martinelli et al., Nucl. Phys. B445 (1995) 81

7. M. Göckeler et al., Nucl. Phys. B (Proc. Suppl.) 47 (1996) 493

8. B. Sheikholeslami and R. Wohlert, Nucl. Phys. B259 (1985) 572

9. R. Sommer, this conference

10. S. Caracciolo, this conference

11. A. Borelli, C. Pittori, R. Frezzotti and E. Gabrielli, Nucl. Phys. B409 (1993) 382

12. R. Horsley, this conference

13. S. Caracciolo, P. Menotti and A. Pelissetto, Nucl. Phys. B375 (1992) 195

14. S. Capitani and G. Rossi, Nucl. Phys. B433 (1995) 351

15. M. Göckeler et al., Nucl. Phys. B (Proc. Suppl.) 42 (1995) 337

16. M. Göckeler et al., DESY preprint DESY 96031 (hep-lat/9602029), to be published in Phys. Rev. D 